\title{
Age-related differences in executive control of working memory
}

\author{
ROEE HOLTZER \\ Columbia University College of Physicians and Surgeons, New York, New York \\ and Albert Einstein College of Medicine, Yeshiva University, New York, New York \\ and \\ YAAKOV STERN and BRIAN C. RAKITIN \\ Columbia University College of Physicians and Surgeons, New York, New York
}

\begin{abstract}
In two experiments, we used dual-task methodology to assess the effect of aging on executive control of working memory. We hypothesized that (1) age-related dual-task costs would be observed even when individual tasks represent different perceptual modalities; (2) age would modulate the effect of increased temporal overlap on dual-task performance; and (3) the vulnerability of specific memory mechanisms to interference would be age related. We found that aging was associated with disproportionate dual-task costs that increased when extending the overlap between individual tasks. The effect of interference with encoding, and arguably output, was disproportionately larger in old than in young individuals. Ensuring that individual tasks represent different perceptual modalities is important but insufficient when using dual-task methodology to assess the effect of aging on executive function. The degree of temporal overlap between individual tasks and the sensitivity of specific memory operations to interference should be considered, as well.
\end{abstract}

Dual-task methodology has been used to evaluate one aspect of executive control-the allocation and monitoring of attentional resources to competing task demands (Baddeley, 1996, 2001; Baddeley \& Hitch, 1974). While the effect of aging on dual-task performance has been studied extensively (for a review, see Hartley, 1992; McDowd \& Shaw, 2000) and evaluated using meta-analytic procedures (Verhaeghen \& Cerella, 2002; Verhaeghen, Marcoen, \& Goossens, 1993; Verhaeghen, Steitz, Sliwinski, \& Cerella, 2003), the findings have been inconsistent. A number of studies have found that aging is associated with increased costs in dual-task performance (e.g., Crossley \& Hiscock, 1992; Li, Lindenberger, Freund, \& Baltes, 2001; Salthouse, Rogan, \& Prill, 1984; Whiting \& Smith, 1997), but others failed to demonstrate this relation (e.g., Nyberg, Nilsson, Olofsson, \& Bäckman, 1997; Somberg \& Salthouse, 1982; Tun \& Wingfield, 1994; Wickens, Braune, \& Stokes, 1987). Moreover, the theoretical basis for those changes remains controversial (Meyer, Glass, Mueller, Seymour, \& Kieras, 2001).

The present experiments were intended to shed light on our understanding of the effect of aging on executive con-

This work was supported in part by Federal Grant T32 AG00261. R. H. is in the Ferkauf Graduate School of Psychology, and in the Department of Neurology at Albert Einstein. Y.S. is affiliated with the Taub Institute and the Departments of Neurology and Psychiatry at Columbia. Correspondence should be addressed to B. C. Rakitin, Cognitive Neuroscience Division of the Taub Institute and Department of Neurology, Columbia University College of Physicians and Surgeons, 630 West 168th Street, P \& S Box 16, New York, NY 10032 (e-mail: br130@columbia.edu). trol of working memory. To accomplish this goal, we designed a dual-task paradigm with specific methodological and theoretical considerations in mind. The two individual tests were selected to represent visual and verbal aspects of working memory. This was done to examine whether using single tasks that represent different perceptual modalities insulates against age-related dual-task costs. In addition, two dual-task conditions were created that differ in the temporal arrangement of the tasks. In the first dual-task condition, overlap was limited to the retention phase of the visual task. In the second dual-task condition, the overlap extended over the encoding, retention, and output phases of the visual task. Executive control of working memory was conceived as the ability to monitor and allocate attentional resources to two competing individual working memory tasks and was measured in terms of the dual-task costs incurred relative to performance on the single tasks. Moreover, comparing performance costs in the two dual-task conditions afforded insight into whether executive control of working memory was differentially taxed in young and old persons when extending the temporal overlap to the encoding and output phases of the visual task. Performance on the verbal task afforded a more direct examination of the sensitivity of specific memory operations to interference.

A series of studies have used dual-task methodology to examine executive control of working memory in normal aging and in people with Alzheimer's disease (Baddeley, Baddeley, Buck, \& Wilcock, 2001; Baddeley, Bressi, Della Sala, Logie, \& Spinnler, 1991; Baddeley, Logie, Bressi, 
Della Sala, \& Spinnler, 1986). The tasks utilized in those studies conformed to an established working memory model (Baddeley, 2001; Baddeley \& Hitch, 1974) in which each single task is presumably processed via a separate slave system (phonological loop, visuospatial sketchpad). Resource allocation to each task/modality is presumed to occur via a third mechanism, the central executive. In these studies, Alzheimer's disease, but not normal aging, produced disproportionate dual-task costs. These findings suggested that the central executive remained intact in old age.

However, in the studies described above the individual tasks were continuous, affording the possibility that subjects interlaced cognitive operations needed to complete the individual tasks, thereby reducing or avoiding direct interference between the tasks. Consequently, age-related dual-task costs may have been underestimated. Careful manipulation of the degree of overlap between individual tasks may help to provide insight into the specific operations underlying dual-task performance in general, and age-related differences in dual-task costs, in particular.

The psychological refractory period (PRP) method represents one approach to manipulating the degree of interference by systematically changing the stimulus onset asynchrony of the two tasks (for a review of this literature, see Pashler, 1994). Using this methodology, Hartley (2001) suggested that age differences in dualtask costs may be confined to concomitant generation and execution of two similar motor programs. Another approach is to limit the overlap to specific cognitive operations within a task. For example, two studies showed that age differences in dual-task costs, as measured by central tendency (Anderson, Craik, \& Naveh-Benjamin, 1998) and dispersion parameters (Anderson, 1999) of reaction time, varied depending on whether the interference occurred during encoding (i.e., stimulus presentation), recall, or recognition (i.e., probe presentation) components of a working memory task. The individual tasks in these studies were not designed to tap the resources of separate slave system/modalities, a point that is relevant to the present investigation.

Three important issues warrant clarification with respect to our understanding of executive control of working memory in aging. Whereas the deleterious effect of old age on dual-task performance has been reported in a large body of literature, it remains an open question whether this robust aging effect is eliminated or retained when each single working memory task represents a different slave system/modality. Moreover, it is of considerable interest to examine whether the degree of temporal overlap between such tasks has a differential effect on agerelated dual-task performance. A related issue is whether interference with specific memory mechanisms differentially affects performance of young and old individuals.

\section{Present Study}

Experiment 1 was conducted to evaluate the following three hypotheses concerning the effect of aging on exec- utive control of working memory. First, we hypothesized that age-related dual-task costs would be observed in a novel dual-task working memory paradigm in which the two single tasks were designed to represent the phonological loop and visuospatial sketchpad. That is, we assessed whether separate perceptual modalities insulated against the effect of aging on dual-task performance. The dual-task paradigm was composed of a delayed visual recognition (DVRT) task and an auditory computerized digit span (CDS) task.

Second, we hypothesized that increased temporal overlap between the two single tasks would exacerbate the effect of aging on dual-task performance. Two dual-task conditions varied in terms of the degree of overlap between the two single tasks (see Figure 1). In the partial interference condition, the digit span task overlapped only with the retention phase of the delayed visual recognition task. In the complete interference condition, two digit sets were administered so that the overlap was extended over the encoding, retention, and output mechanisms of the DVRT. Comparing the DVRT performance between the two dual-task conditions addressed the questions of whether manipulating the degree of temporal overlap produced differential dual-task costs and whether these costs were differentially related to age. The results of this analysis will help determine the extent to which the results of previous experiments depended on the use of continuously overlapping tasks.

Third, we hypothesized that the sensitivity of encoding and output to interference would be modulated by aging. Comparing digit recall accuracy between the two dualtask conditions afforded insight regarding this question. In the partial interference condition, the encoding and output of the digit span task were performed during the retention phase of the DVRT. Hence, interference was limited to the retention of visual stimuli. In the complete interference dual-task condition, encoding of the first digit set was concomitant with the encoding of the visual stimuli, but digit recall occurred during the retention phase. Hence, interference with the encoding of digits varied between the two dual-task conditions, whereas the recall of digits remained constant. If increased age differences are observed in the complete interference (CI) condition relative to the partial interference (PI) condition, it would suggest that the susceptibility of encoding to interference is age related. The second digit set of CI was encoded during the second half of the retention phase, but repetition of the digits was concurrent with the DVRT output. In comparison with PI, encoding demands were similar, but the interference with the output of the digit span task varied. Increased age-related differences in digit recall in the second digit set of the CI task relative to the PI task would suggest that the consequence of interfering with memory output is age related.

Experiment 2 was designed to reevaluate the same hypotheses while addressing methodological limitations that were inherent in the first experiment. 


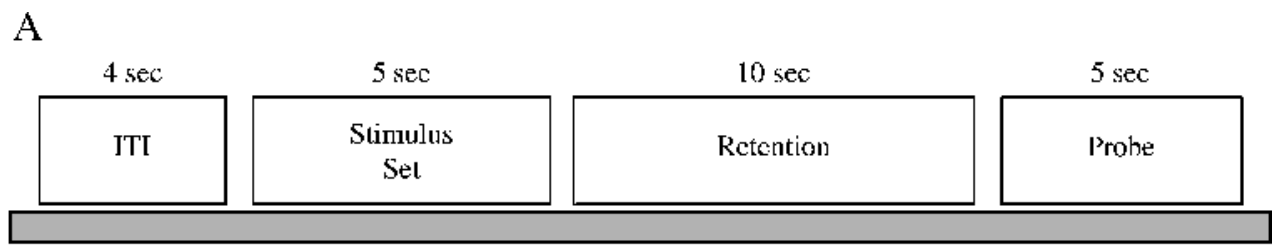

B

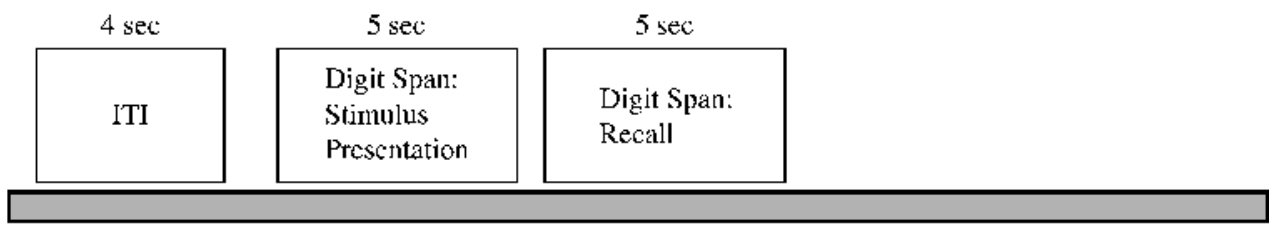

C.

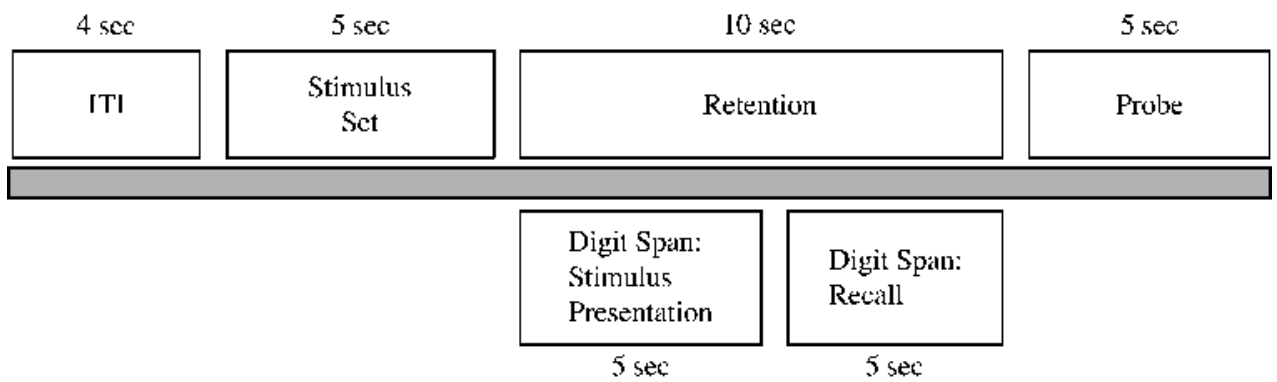

D

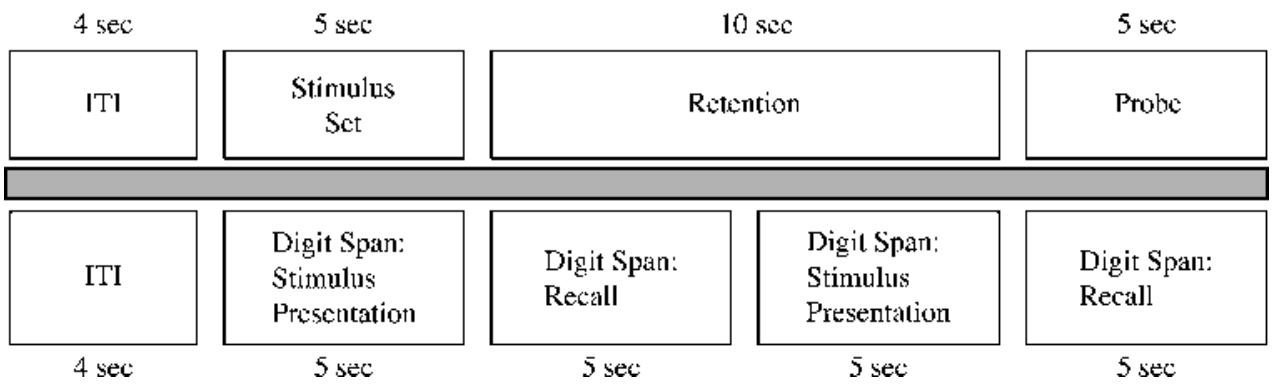

Figure 1. Schematic presentation of the delayed visual recognition task (DVRT) and the computerized digit span (CDS) task in single- and dual-task conditions. (A) DVRT alone. (B) CDS task alone. (C) Dualtask partial interference. (D) Dual-task complete interference.

\section{EXPERIMENT 1}

\section{Method}

\section{Participants}

Sixteen older adults, ages $65-85(M=74.3, S E=1.4)$, and 16 younger adults, ages $19-31(M=21.2, S E=0.76)$, participated in the present study. Within each age group, $62.5 \%$ were females. The old participants were community residents who were recruited from newspaper advertising and senior centers, and who have previously participated in studies conducted at the Columbia University Medical Center. The young participants were undergraduate students at Columbia University. All the participants were determined to be in good health, based on self-report and clinical interview. Exclusionary criteria were medical and psychological history that might affect cognition (e.g., brain trauma, neurodegenerative diseases, depression), medications known to have an effect on test performance, and history of learning disability. All the participants provided informed consent, and all were compensated for their participation.
The young and old participants were comparable on a number of demographic variables and neuropsychological screens of cognitive and intellectual function. Dementia Rating Scale (DRS; Mattis, $1988)$ total scores for the young $(M=142, S E=.38)$ and old $(M=$ $141, S E=.94)$ participants were comparable and at least $1 S D$ above the recommended dementia cutoff score of 123 (Mattis, 1988). The National Adult Reading Test (NART; Nelson, 1982) mean scores for the young $(M=39.3, S E=1.1)$ and old $(M=39.9$, $S E=1.7$ ) participants were comparable and indicative of estimated verbal intellectual function that was, on average, $1 S D$ above the mean. The difference in years of education for the young $(M=$ $14.8, S E=.36)$ and old $(M=15.9, S E=.39)$ participants was not statistically significant.

\section{Apparatus}

A Macintosh iBook computer with a 12.1-in. diagonal viewable monitor was used to administer the single- and dual-task conditions. A delayed visual recognition procedure and an auditory digit recall task 
were implemented using PsyScope software (Cohen, MacWhinney, Flatt, \& Provost, 1993). Visual stimuli were presented on the computer screen. Keys on the left and right sides of the keyboard served as response keys for the visual task. Auditory stimuli for the digit span test were generated by the computer and amplified by digital speakers. The timing of participants' verbal responses on the digit span task was monitored using a headset microphone that was connected to the computer via the voice-key port of the PsyScope button box. The computer registered the time when each digit was produced.

\section{Procedures}

All training and testing procedures were conducted in the same laboratory room in the medical center. Each participant was tested individually. The single- and dual-task conditions are presented schematically in Figure 1.

Delayed visual recognition task (Figure 1A). The delayed visual recognition procedure is a nonverbal adaptation of the Sternberg task (Sternberg, 1966, 1969). It consisted of 60 trials, divided into five 12-trial blocks. Each trial consisted of an encoding, retention, and probe phase. In the encoding phase, two or four visual stimuli were presented for $5 \mathrm{sec}$. This was followed by a 10 -sec retention interval during which the computer screen was blank. Then, a single probe item was presented for $5 \mathrm{sec}$. The probe item either matched (true positive) or did not match (true negative) one of the stimuli from the study set. The participants indicated whether the probe item was included in the initial set by a differential buttonpress. The participants were instructed to respond as quickly as possible. Assignment of response keys to the true positive and true negative conditions was counterbalanced across participants within each age group. Each study block had an equal number of the four trial types - that is, set size 2 and 4, and positive and negative probes. Their order was randomly distributed.

The DVRT was run twice as a single task. In the first run, designated as the training procedure, accuracy feedback with respect to the probe decision was given after each trial. The second run, referred to as the alone/baseline condition, omitted the feedback and was intended to serve as a baseline comparison to the dual tasks.

Twelve Microsoft Word symbol characters (e.g., $\xi$, ), $\varphi, \wp$ ) served as stimuli for the DVRT. Sixty study sets of stimuli with associated probes were generated for each run of the task. Two additional 24 study sets with associated probes were generated for the training procedures of the two dual-task conditions.

Computerized digit span (Figure 1B). This task consisted of auditory presentation and verbal recall of a five-digit set. The participants listened to a set of five numbers, produced by the computer at a rate of one digit per second. They were then asked to repeat the digits in the same order and at the same pace. The times at which each digit was produced was monitored by the computer. The accuracy of the digit recall was hand-recorded by the tester.

Digital recordings of a female voice were used to present the stimuli (digits 1-9). The average presentation duration per digit was $465 \mathrm{msec}$. Randomization within each digit set was constrained by two factors: Digits presented one after the other could not be consecutive, either in descending or ascending order; and identical pairs of digits were not presented in consecutive digit sets.

The training task, consisting of 25 five-digit sets, was used to train the participants to comply with the rate and accuracy parameters of the task. A visual cue (asterisk) was used to help the participants recall the digits at a constant pace of $1 \mathrm{sec}$ per digit. At the end of each trial, a message appeared on the computer screen indicating whether the digit recall had been completed within the 5-sec time allotted. The tester provided feedback on the accuracy of the digit recall.

The CDS testing was administered immediately after the training task. It also consisted of 25 digit sets. The visual cue and accuracy feedback used during training were removed from the CDS testing condition that served as a baseline comparison to the two dual tasks.

Training for the two dual tasks consisted of 24 trials per condition. At the end of each trial, feedback was provided on the computer screen, indicating whether the probe decision was accurate and whether the digit recall was completed within the time allotted. Also, the tester provided feedback on the accuracy of the digit recall.

Dual-task partial interference (Figure 1C). In the first dual task, the overlap between the two tasks was limited to the retention period of the DVRT. After the encoding period of the DVRT, the participants were required to attend to and repeat one set of five digits during the $10-\mathrm{sec}$ retention interval, prior to the appearance of the DVRT probe. The DVRT probe then appeared, and the participants responded to that probe. There were 60 trials of this dual condition, run in same manner as the DVRT alone.

Dual-task complete interference (Figure 1D). In the second dual task, overlap of the two tasks was complete. The participants were required to encode the visual study set while listening to the first set of digits, recall the digits during the first $5 \mathrm{sec}$ of the retention interval, attend to a second set of digits during the last $5 \mathrm{sec}$ of the retention interval, and then recall the digits while making a decision about the identity of the probe. Two five-digit sets were administered during each trial, amounting to a total of 120 sets for the entire dual-task condition. It is important to note that while the degree of overlap between the two tasks was extended during the dual-task complete interference, the single visual and verbal tasks remained unchanged. The participants were instructed that the visual and verbal tasks were equally important.

\section{Neuropsychological Measures}

Two measures were administered to ensure that current and premorbid levels of intellectual functioning were within normal limits.

NART (Nelson, 1982) is a reading test of 50 irregularly spelled words that is considered to be a measure of premorbid ability because it relies more on previously acquired knowledge than on current cognitive function. It correlates highly with measures of general intelligence and is used to estimate IQ while taking education and gender into account (Spreen \& Strauss, 1998).

DRS (Mattis, 1988) is a widely used scale that examines five areas (attention, initiation and perseveration, construction, conceptualization, memory) that are sensitive to the cognitive changes associated with dementia of the Alzheimer's type. The maximal total score in the DRS is 144, and the suggested dementia cutoff score is 123 (Mattis, 1988).

The Digit Span Test (Wechsler Adult Intelligence Scale-3rd Ed. [WAIS-III]; Wechsler, 1997) is the most commonly used test for measuring the span of verbal recall. It was administered according to standardized procedures to ensure that performance on this measure was within acceptable age norms.

\section{Procedure}

The first and second experimental sessions for each participant were conducted at the same time of day. Once written consent was obtained at the beginning of the first session, the tester conducted a comprehensive interview to obtain demographic, medical, and psychological information, ensuring compliance with the inclusion and exclusion criteria of the study. Subsequently, the training components of the DVRT, NART, DRS, and alone/baseline condition of the DVRT were administered. The Digit Span Test (WAIS-III) and training and testing of the CDS concluded the first session.

In order to proceed to the second day of testing, the participants were required to recall the digit sets at a minimum of $75 \%$ accuracy and comply with the rate at which the digits had to be repeated. Minimum accuracy for the DVRT was set at $75 \%$. Two old participants and 1 young participant were excluded from the study due to failure to comply with the performance criteria of the digit recall task.

On the second day, the participants received 12 trials of the single DVRT (training version) to refamiliarize them with the task. They were also administered 10 additional CDS trials to ensure compliance with the accuracy and response rate indices of the task. The PI and CI dual tasks were then administered in a random order 
to ensure that each task was administered first to $50 \%$ of the participants in each age group. In each case, the 60 test trials of the PI and CI dual tasks were administered after completion of their respective training procedures.

\section{Statistical Analyses}

For all analyses in this article, the level of statistical significance was set at $p=.05$. Tests for sphericity were carried out in each analysis. Huynh-Feldt corrected significance levels are reported for any effect for which the sphericity test was significant. Helmert planned contrast analyses were carried out on significant main effects of the within-subjects variables and their interactions with age. These contrast analyses served to accomplish two goals: (1) examine differences between the alone condition and the dual tasks (treated as a construct), and (2) directly compare the two dual-task conditions to evaluate the effect of manipulating the degree of temporal overlap between tasks and the sensitivity of encoding and output to interference.

The issue of whether the dual-task costs were disproportionate to group differences at baseline was examined for the reaction time and accuracy measures of the DVRT. The advantages and disadvantages of methods that seek to address the issue of proportionality in evaluating dual-task costs have been discussed with respect to proportional transformation and the Brinley plot (e.g., Fisk \& Fisher, 1994; Hartley, 2001; Perfect, 1994) and log transformation (Baddeley et al., 2001). In the present study, proportional transformation was used for the reaction time and accuracy measures of the DVRT.

DVRT reaction time. Median reaction time was derived from correct responses only for each participant and served as the dependent measure. First, inferential statistics were performed using a repeated measures analysis of variance (ANOVA) on the raw data. The within-subjects variables were the three-level task condition (DVRT alone, PI, CI), two-level probe type, and two-level set size. Age served as the between-groups variable. Second, a proportional transformation measure of reaction time was derived for the PI and $\mathrm{CI}$ conditions, per participant, using the following equation: (Mdn dual task - Mdn single task)/Mdn single task. Then, a second repeated measures ANOVA was run with the two ratio changes in reaction time serving as dependent measures. The within-subjects factors were the two-level task condition, set size, and probe type. Age was the between-subjects factor.

DVRT accuracy data. The total number of correct responses for the single-task and two dual-task conditions served as dependent measures. In keeping with the reaction time analyses, a two- phase repeated measures ANOVA was used. First, data were analyzed using the raw accuracy data. The within-subjects variables were the three-level task condition (DVRT alone, PI, CI), two-level probe type, and two-level set size. Age served as the betweengroups variable. Second, a proportional transformation measure of accuracy was derived, per participant, for the PI and CI tasks using the following equation: (total correct dual task - total correct single task)/(total correct single task). The two ratio changes in accuracy for the PI and CI conditions served as dependent measures. The within-subjects factors were the two-level task condition, twolevel probe type, and two-level set size. Age was the betweensubjects factor.

CDS. The percentage of inaccurate digits recalled was calculated for each task. In the alone condition, the total possible number of digit errors was 125 . In the PI, and in each CDS set of the CI, the total number of possible digit errors was 300 .

Two different repeated measures ANOVAs were performed to evaluate whether the accuracy of digit recall varied as a function of task interference (three-level within-subjects variable) and age (betweensubjects factor). The first ANOVA included the CDS task alone, PI, and the first digit set of the CI. The second repeated measures ANOVA included the second digit set of the CI as the third level task. These two models were designed to assess the sensitivity of encoding (Model 1) and output (Model 2) to interference. Statistical analyses of the CDS performance were limited to digit recall accuracy. Compliance with the pace and 5-sec time interval at which digits were repeated was first achieved by the training procedures of both the single and dual tasks and then ascertained behaviorally at the testing procedures by the tester.

\section{Results and Discussion}

\section{DVRT}

Reaction time: Raw data. Descriptive statistics $(M$, $S E$ ) for the raw and transformed reaction time data in the alone, PI, and CI conditions are presented per group in Table 1 . The data were analyzed by means of repeated measures ANOVAs with task (three-level), set size (twolevel), and probe identity (two-level) as the within-subjects variables and age as the between-subjects factor. The main effects were significant for age $[F(1,30)=58.898$, $p<.001], \operatorname{task}[F(2,60)=9.715, p<.001]$, and set size

Table 1

Descriptive Statistics $(M, S E)$ for Reaction Times (RTs, in Milliseconds) and the Change Ratio in RT per Group for the Alone, PI, and CI Conditions of the DVRT

\begin{tabular}{|c|c|c|c|c|c|c|c|c|}
\hline & \multicolumn{4}{|c|}{ True Negative } & \multicolumn{4}{|c|}{ True Positive } \\
\hline & \multicolumn{2}{|c|}{ Set Size 2} & \multicolumn{2}{|c|}{ Set Size 4} & \multicolumn{2}{|c|}{ Set Size 2} & \multicolumn{2}{|c|}{ Set Size 4} \\
\hline & $M$ & $S E$ & $M$ & $S E$ & $M$ & $S E$ & $M$ & $S E$ \\
\hline \multicolumn{9}{|l|}{ Alone } \\
\hline Young raw & 921 & 55 & 1,046 & 40 & 970 & 73 & 1,074 & 52 \\
\hline Old raw & 1,270 & 45 & 1,693 & 140 & 1,309 & 58 & 1,760 & 110 \\
\hline \multicolumn{9}{|l|}{ PI } \\
\hline Young raw & 708 & 39 & 897 & 75 & 729 & 55 & 876 & 75 \\
\hline Young $\Delta$ ratio & -.204 & .05 & -.155 & .07 & -.193 & .08 & -.180 & .05 \\
\hline Old raw & 1,219 & 109 & 1,441 & 62 & 1,316 & 67 & 1,543 & 116 \\
\hline Old $\Delta$ ratio & -.052 & .06 & -.162 & .11 & .026 & .07 & -.125 & .04 \\
\hline \multicolumn{9}{|l|}{$\mathrm{CI}$} \\
\hline Young raw & 810 & 75 & 835 & 47 & 681 & 40 & 828 & 72 \\
\hline Young $\Delta$ ratio & -.049 & .12 & -.205 & .06 & -.236 & .07 & -.222 & .06 \\
\hline Old raw & 1,265 & 104 & 1,460 & 102 & 1,545 & 77 & 1,717 & 104 \\
\hline Old $\Delta$ ratio & -.016 & .05 & -.164 & .11 & .217 & .08 & .006 & .05 \\
\hline
\end{tabular}

Note $-n=16$ for each group. 
$[F(1,30)=52.475, p<.001]$. Overall, as expected, old participants had slower reaction times compared with young participants across the three tasks. Interpretations of the effects of task and set size are better understood in light of the significant two- and three-way interactions delineated below. The two-way interaction of task and age was statistically significant $[F(2,60)=3.620, p<$ $.05]$. Helmert planned contrast analysis, carried out on the task $\times$ age group interaction, showed that the difference in reaction time between young and old participants increased significantly between the PI and CI conditions $[F(1,30)=6.940, p<.05]$. Group differences between the dual-task condition (treated as a construct) and the single-task condition were not significant.

The two-way interaction of age $\times$ probe identity $[F(1,30)=4.622, p<.05]$ and three-way interaction of age $\times$ task $\times$ probe identity $[F(2,60)=4.297, p<.05]$ were significant. These results show that while young participants responded more quickly to true positives than to true negatives in the dual-task conditions, old participants showed the opposite response pattern. Helmert planned contrast analyses, carried out on the age $\times$ task $\times$ probe identity interaction, revealed that age differences in reaction time to probe identity were larger in the CI condition relative to the PI condition $[F(1,30)=8.280$, $p<.01]$. Age differences in reaction time to the probe identity between the dual-task condition (treated as a construct) and the single-task condition were not significant.

The two-way interaction of set size $\times$ task $[F(2,30)=$ 4.404, $p<.05$ ] and three-way interaction of set size $\times$ task $\times$ age $[F(2,60)=4.491, p<.05]$ were significant. Whereas increased age differences in reaction time in the $\mathrm{PI}$ and CI conditions relative to the alone condition were observed for both set sizes, they were more noticeable for set size 2. Helmert planned contrast analysis, carried out on the three-way interaction, showed that age difference in reaction time increased significantly in the dualtask conditions relative to the DVRT when performed alone $[F(1,30)=5.708, p<.05]$. Age differences between the PI and CI were not significant. No other main effects or interactions were significant.

Reaction time: Proportional transformation. The data were analyzed by means of a repeated measures ANOVA with task (two-level), set size (two-level), and probe identity (two-level) as the within-subjects variables and age as the between-subjects factor. The dependent measures were mean ratio changes in reaction time in the PI and CI conditions. The main effects were significant for age $[F(1,30)=4.249, p<.05]$ and task $[F(1,30)=4.829, p<.05]$. However, the age $\times$ task interaction was marginal $[F(1,30)=3.929, p=.057]$. The two-way interaction of task $\times$ size $[F(1,30)=5.293$, $p<.05]$ and three-way interaction of age $\times$ task $\times$ probe type $[F(1,30)=7.476, p<.05]$ were statistically significant. The remaining main effects and interactions were not significant.

Reaction time: Summary. Analyses of raw reaction time data showed that, as expected, old participants were slower across the three tasks and that age differences increased in the dual-task conditions relative to the DVRT when performed alone. In addition, set size and probe type modulated the increase in age-related dual-task costs. Age differences remained significant when analyses were run on the transformed data, indicating that the costs incurred by old participants were disproportionate to group differences at baseline. However, age differences between the PI and CI conditions were not reliable when analyses were run on the transformed reaction time data. Finally, an important caveat is that the age differences in the dual-task reaction time costs are due to greater improvement in performance by young compared with old participants in the dual-task relative to the single-task condition.

Accuracy: Raw data. Descriptive statistics $(M, S E)$ for the total correct responses and proportional change in accuracy in the PI and CI conditions relative to baseline

Table 2

Descriptive Statistics $(M, S E)$ for Total Correct Responses and the Change Ratio in Correct Responses per Group for the Alone, PI, and CI Conditions of the DVRT

\begin{tabular}{|c|c|c|c|c|c|c|c|c|}
\hline & \multicolumn{4}{|c|}{ True Negative } & \multicolumn{4}{|c|}{ True Positive } \\
\hline & \multicolumn{2}{|c|}{ Set Size 2} & \multicolumn{2}{|c|}{ Set Size 4} & \multicolumn{2}{|c|}{ Set Size 2} & \multicolumn{2}{|c|}{ Set Size 4} \\
\hline & $M$ & $S E$ & $M$ & $S E$ & $M$ & $S E$ & $M$ & $S E$ \\
\hline \multicolumn{9}{|l|}{ Alone } \\
\hline Young TC & 14.9 & .06 & 14.6 & .15 & 14.8 & .14 & 14.7 & .15 \\
\hline Old TC & 14.9 & .08 & 13.7 & .34 & 14.1 & .38 & 13.3 & .45 \\
\hline \multicolumn{9}{|l|}{ PI } \\
\hline Young TC & 14.8 & .10 & 13.9 & .36 & 14.5 & .20 & 14.1 & .27 \\
\hline Young $\Delta$ ratio & -.008 & .01 & -.046 & .02 & -.016 & .01 & -.042 & .02 \\
\hline Old TC & 14.5 & .22 & 13.8 & .36 & 12.4 & .55 & 10.8 & .80 \\
\hline Old $\Delta$ ratio & -.024 & .01 & .013 & .03 & -.102 & .04 & -.177 & .05 \\
\hline \multicolumn{9}{|l|}{ CI } \\
\hline Young TC & 14.8 & .11 & 14.6 & .12 & 14.3 & .24 & 12.8 & .52 \\
\hline Young $\Delta$ ratio & -.012 & .01 & .001 & .01 & -.028 & .02 & -.130 & .04 \\
\hline Old TC & 14.1 & .22 & 12.6 & .43 & 11.6 & .72 & 9.1 & .84 \\
\hline Old $\Delta$ ratio & -.050 & .01 & -.069 & .03 & -.166 & .05 & -.310 & .06 \\
\hline
\end{tabular}


are presented per group in Table 2. The accuracy data were analyzed by means of a repeated measures ANOVA with task (three-level), probe identity (two-level), and set size (two-level) as the within-subjects variables and age as the between-subjects factor. Because the probe identity and set size factors did not interact (i.e., three-way interaction) with age and task conditions, the repeated measures ANOVA was rerun, collapsing over probe identity and set size. The main effects were significant for age $[F(1,30)=34.117, p<.001]$ and task $[F(2,60)=$ $26.658, p<.001]$. These results revealed that accuracy was reduced as a function of old age and task condition. Furthermore, the two-way interaction of age $\times$ task was significant $[F(2,60)=7.596, p<.01]$. Helmert planned contrast analysis was carried out on the age $\times$ task interaction, revealing that differences in accuracy between young and old participants increased significantly in the dual-task condition compared with the DVRT alone $[F(1,30)=10.245, p<.01]$. Moreover, age differences in accuracy were significantly larger in the CI compared with the PI $[F(1,30)=4.683, p<.05]$.

Accuracy: Proportional transformation. The data were analyzed by means of a repeated measures ANOVA with task (two-level) as the within-subjects variable and age as the between-subjects factor. Dependent measures were mean accuracy changes in the PI and CI relative to the alone task. The main effects were significant for age $[F(1,30)=10.692, p<.01]$ and task $[F(1,30)=11.586$, $p<.01]$. The age $\times$ task interaction was significant $[F(1,30)=5.281, p<.05]$.

Accuracy: Summary. Accuracy decreased as a function of age and task interference. Age-related differences in accuracy were disproportionately larger in the dualtask condition relative to the alone condition with old participants showing reduced accuracy due to interference. Furthermore, a direct comparison between the PI and CI conditions showed that increased overlap between the individual tasks was more deleterious to old than to young participants.

Table 3

CDS-Percentage of Incorrect Digit Recall $(M, S E)$ per Group in the Alone, PI, and CI Conditions

\begin{tabular}{lccccc}
\hline & \multicolumn{2}{c}{ Young } & & \multicolumn{2}{c}{ Old } \\
\cline { 2 - 3 } \cline { 5 - 6 }$M$ & $S E$ & & $M$ & $S E$ \\
\hline Alone & .95 & .41 & & 3.3 & .50 \\
PI & .74 & .25 & & 4.18 & .69 \\
$\quad$ Set size 2 & .54 & .22 & & 4.21 & .96 \\
$\quad$ Set size 4 & .95 & .37 & & 4.16 & .85 \\
CI & & & & \\
$\quad$ 1st digit set & 1.90 & .47 & 9.54 & 1.21 \\
$\quad$ Set size 2 & 1.75 & .55 & 9.58 & 1.37 \\
$\quad$ Set size 4 & 2.08 & .67 & 9.50 & 1.26 \\
CI & & & & \\
$\quad$ 2nd digit set & 2.04 & .47 & 8.35 & 1.30 \\
$\quad$ Set size 2 & 1.87 & .40 & 7.71 & 1.22 \\
$\quad$ Set size 4 & 2.20 & .62 & 9.08 & 1.49 \\
\hline
\end{tabular}

Note-In the PI and CI conditions, digit recall performance is also provided as a function of DVRT set size. $n=16$ for each group.

\section{CDS}

Descriptive statistics for the percentage of incorrect digit recall per group in the alone, PI, and CI dual-task conditions are presented in Table 3. Repeated measures ANOVAs were performed on two models with age as the between-subjects factor and task as the three-level withinsubjects variable. The first model included the percentage of incorrect digit recall in the alone and PI and the first digit set of the CI. The second model used the second digit set of the $\mathrm{CI}$ as the third-level task.

Model 1. The main effects for age $[F(1,30)=38.575$, $p<.001]$, task $[F(2,60)=29.591, p<.001]$, and the age $\times$ task interaction $[F(2,60)=14.897, p<.001]$ were statistically significant. Helmert planned contrast analyses carried out on the age $\times$ task interaction revealed that the difference between young and old participants was significantly larger in the dual-task condition compared with the alone condition $[F(1,30)=16.738$, $p<.01]$. Moreover, group differences were significantly larger in the CI compared with the PI $[F(1,30)=13.796$, $p<.01]$. These findings provided direct evidence of the vulnerability of encoding to interference in old age.

Model 2. Similar to the first model, the main effects for age $[F(1,30)=30.451, p<.001]$, task $[F(2,60)=17.295$, $p<.001]$, and the age $\times$ task interaction $[F(2,60)=$ $6.589, p<.001]$ were significant. Helmert planned contrast analyses carried out on the age $\times$ task interaction showed that the difference between young and old participants was significantly larger in the dual-task condition relative to the alone condition $[F(1,30)=14.616$, $p<.01]$. Moreover, age differences were significantly larger in the CI compared with the PI $[F(1,30)=4.313$, $p<.05]$. These findings were suggestive of age-related sensitivity to interference with output operations.

CDS performance: Summary. Digit recall accuracy was reduced as a function of age and interference. Old participants incurred dual-task costs that were significantly larger than those shown by young participants. Moreover, compared with the PI, old participants demonstrated a greater reduction in digit recall accuracy in both the first and second digit sets of the CI than that shown by young participants. These findings provided evidence of the sensitivity of encoding and arguably output to interference in aging.

Experiment 1 was designed to evaluate three hypotheses with respect to the effect of aging on dual-task performance. First, it was of interest to examine whether age-related dual-task costs were observed in a paradigm in which the two individual working memory tasks were designed to represent the phonological loop and visuospatial sketchpad systems. Second, we assessed whether the extent of temporal overlap between the individual tasks modulated the effect of aging on dual-task performance. Third, comparison of the CDS performance in the PI and CI conditions was used to evaluate whether the sensitivity of encoding and output to interference was related to age. We found that age-related dual-task costs were present although the individual tests were 
across two perceptual modalities. The extent of temporal overlap between individual tasks and interference with encoding and output operations modulated the negative effect of aging on dual-task performance.

However, conclusions were limited by several methodological concerns. The two dual-task conditions were administered after the alone condition. This design was intended to simplify the training for the dual-task conditions, but as a result it could be argued that the effects of aging on dual-task performance in Experiment 1 were due to order or effects brought about by practice. The age-related dual-task costs in reaction time were attributed to young participants' faster performance in the PI and $\mathrm{CI}$ conditions relative to the alone condition, which suggested that the order effect might have been related to age. Furthermore, this facilitation effect was a counterintuitive finding. Lastly, the limited number of visual stimuli (12) in the DVRT constituted a limitation for a task that was designed to represent the visuospatial sketchpad. It is possible that repeated presentations led to phonologic processing of those stimuli.

\section{EXPERIMENT 2}

Experiment 2 was designed to address the above methodological issues. The two dual-task conditions (PI and CI) were preserved, but several important changes were made. The visual stimuli of the DVRT were replaced by 450 different computer-generated closed-curve shapes. Each shape was presented only once in the testing conditions of each participant. This presented an advantage in that both the novelty and appearance of the shapes maximized visual demands and limited the extent of phonologic processing. The number of trials in the testing conditions of the DVRT was reduced to 40 to ensure that the entire experimental protocol could be completed in one session. The training procedures for the CDS and DVRT in the single- and dual-task conditions were completed first. However, the administration order of the four test conditions (CDS alone, DVRT alone, PI, CI) was randomized to address the order effect in Experiment 1.

\section{Method}

\section{Participants}

Sixteen older adults, ages 65-80 $(M=73, S E=1.2)$, and 16 younger adults, ages $19-30(M=23.4, S E=.73)$, participated in Experiment 2. Gender distribution within the young $(43.75 \%$ female) and old (50\% female) groups was comparable. The old participants were community residents who were recruited from newspaper advertising and senior centers, and who have previously participated in studies conducted at the medical center. The young participants were community residents and undergraduate students from local universities who were recruited from newspaper and Internet advertising. Mean education, in years, for the young $(M=$ $15.3, S E=.29)$ and old $(M=14.8, S E=.57)$ participants was comparable. Mean DRS total scores for the young $(M=142.8$, $S E=.43)$ and old $(M=142.3, S E=.51)$ groups were not statistically different and well above the suggested dementia cutoff score. Estimated verbal IQ (NART) was comparable and above average for the young $(M=38.9, S E=2.0)$ and old $(M=39.2, S E=2.3)$ groups. All the participants were determined to be in good health based on self-report and clinical interview. Exclusionary criteria were the same as those applied in Experiment 1. All participants provided informed consent and all were compensated for their participation.

\section{Apparatus and Materials}

The apparatus and materials were similar to those in Experiment 1, except for the visual stimuli of the DVRT. In the present experiment, 450 computer-generated shapes replaced the 12 Microsoft Word symbol characters that were used in the first experiment. These closed-curve shapes were thought to be nonverbal to the extent that they did not correspond to or intuitively relate to real words. One hundred fifty shapes were used for training, and 300 shapes were used for the three testing conditions (DVRT alone, PI, CI). Whereas the complexity of the shapes varied, the shapes in the study set and probe within each trial were equated in terms of their mean pixel ratio. Positive and negative trials were randomly assigned to each test condition with the constraint that trials in each condition were matched in terms of their mean pixel ratio. Examples of trials that vary in terms of their mean pixel ratio are presented in the Appendix.

\section{Procedures}

Training. Training on the CDS and DVRT was similar to that in Experiment 1. However, in contrast to the first experiment, the training procedures for both the single- and dual-task conditions were completed in one session and prior to testing.

Testing. The number of trials in the alone, PI, and CI conditions was reduced from 60 to 40 to shorten the administration time. Each task was divided into four 10-trial blocks. Each study block had an equal number of true positive and true negative probes. Due to the complexity of the shapes, the study set in the present experiment consisted of two shapes only.

The CDS task remained unchanged, except that the number of digit sets was reduced to 40 and 80 in the PI and CI conditions, respectively, to parallel the reduction of trials in the DVRT.

There were four testing conditions: CDS alone, DVRT alone, PI, and CI. Of 24 test order possibilities, 16 were randomly selected with the constraint that each task had to be represented four times in the first, second, third, and fourth order positions. Within each age group, each participant received a different test order. The participants' number (1-16) determined the test order they received. Old and young participants with the same number received the same test order.

\section{Procedure}

In contrast to Experiment 1, all experimental procedures were completed in one session. Once written consent was obtained, the tester conducted a comprehensive interview to gather demographic, medical, and psychological information, ensuring compliance with the inclusion and exclusion criteria of the study. Subsequently, the DRS, NART, and digit span tests were administered. The training procedures for the CDS and DVRT in the single- and dual-task conditions concluded the first part of the experimental session. Then the participants received a 20-min break that was followed by the testing procedures. Similar to Experiment 1, minimum accuracy criteria for the CDS and DVRT in the alone condition were set at $75 \%$. Three old and 1 young participant were excluded from the study due to failure to comply with the above performance criteria.

\section{Results and Discussion}

The statistical procedures in Experiment 2 paralleled those used in the first experiment.

\section{DVRT}

Reaction time: Raw data. Descriptive statistics $(M$, $S E$ ) for the raw and transformed reaction time for the alone, PI, and CI conditions are presented per group in 
Table 4

Descriptive Statistics $(M, S E)$ for Reaction Times (RTs, in Milliseconds) and the Change Ratio in RT per Group for the Alone, PI, and CI Conditions of the DVRT

\begin{tabular}{lccccr}
\hline & \multicolumn{2}{c}{ True Negative } & & \multicolumn{2}{c}{ True Positive } \\
\cline { 2 - 3 } \cline { 5 - 6 } & $M$ & $S E$ & & $M$ & $S E$ \\
\hline Alone & & & & \\
$\quad$ Young raw & 1,236 & 104 & & 1,220 & 80 \\
$\quad$ Old raw & 1,703 & 124 & & 1,750 & 121 \\
PI & & & & \\
Young raw & 1,094 & 79 & & 1,035 & 46 \\
$\quad$ Young $\Delta$ ratio & -.088 & .04 & & -.127 & .04 \\
Old raw & 1,707 & 104 & & 1,535 & 81 \\
Old $\Delta$ ratio & .036 & .06 & & -.092 & .05 \\
CI & & & & \\
Young raw & 1,185 & 117 & & 1,190 & 91 \\
Young $\Delta$ ratio & -.027 & .06 & & -.017 & .05 \\
Old raw & 2,191 & 130 & & 1,997 & 107 \\
Old $\Delta$ ratio & .336 & .07 & .210 & .09 \\
\hline
\end{tabular}

Note $-n=16$ for each group.

Table 4. The data were analyzed by means of a repeated measures ANOVA with task (three-level) and probe identity (two-level) as the within-subjects variables and age as the between-subjects factor. Because the probe identity factor did not interact with age and task, the repeated measures ANOVA was rerun, collapsing over true positive and true negative responses. Main effects were significant for age $[F(1,30)=33.020, p<.001]$ and task $[F(2,60)=15.139, p<.001]$. As expected, old participants had slower reaction times compared with young participants across the three tasks. The two-way interaction of task and age was statistically significant $[F(2,60)=$ $8.025, p<.001]$. Helmert planned contrast analysis, carried out on the task $\times$ age group interaction, showed that the difference in reaction time between young and old participants increased significantly from the alone to the dual-task conditions (treated as a construct) $[F(1,30)=$ 5.687, $p<.05]$. Moreover, age difference in reaction time increased significantly from the PI condition to the CI condition $[F(1,30)=10.857, p<.01]$.

Reaction time: Proportional transformation. The data were analyzed by means of a repeated measures ANOVA with task (two-level) as the within-subjects variable and age as the between-subjects factor. The dependent measures were mean ratio changes in reaction time in the PI and CI conditions. The main effects were significant for age $[F(1,30)=6.697, p<.05]$ and task $[F(1,30)=25.846, p<.001]$. The age $\times$ task interaction was statistically significant $[F(1,30)=7.802, p=.01]$.

Reaction time: Summary. Age-related dual-task costs were reliable and disproportionate to group differences at baseline. Compared with young participants, old participants incurred greater dual-task costs in the CI condition than in the PI condition, which indicated that age modulated the deleterious effect of increasing the temporal overlap between individual tasks.

Accuracy: Raw data. Descriptive statistics $(M, S E)$ for the total correct responses and proportional change in accuracy in the alone, PI, and CI conditions relative to baseline are presented per group in Table 5 . The accuracy data were analyzed by means of a repeated measures ANOVA with task (three-level) and probe identity (two-level) as the within-subjects variables and age as the between-subjects factor. Because the probe identity factor did not interact with age and task, the repeated measures ANOVA was rerun, collapsing over true positive and true negative responses. The main effects were significant for age $[F(1,30)=42.435, p<.001]$ and task $[F(2,60)=49.413, p<.001]$. The two-way interaction of age $\times$ task was significant $[F(2,60)=11.714, p<$ $.001]$. Helmert planned contrast analysis, which was carried out on the age $\times$ task interaction, revealed that differences in accuracy between young and old participants increased significantly in the dual-task condition compared with the DVRT alone condition $[F(1,30)=12.840$, $p<.01]$. Moreover, age differences in accuracy were significantly larger in the CI condition compared with the PI condition $[F(1,30)=10.937, p<.01]$.

Accuracy: Proportional transformation. The data were analyzed by means of a repeated measures ANOVA with task (two-level) as the within-subjects variable and age as the between-subjects factor. The dependent measures were mean ratio changes in accuracy in the PI and $\mathrm{CI}$ conditions. The main effects were significant for age $[F(1,30)=14.851, p<.01]$ and task $[F(1,30)=52.969$, $p<.001]$. The age $\times$ task interaction was significant $[F(1,30)=11.134, p<.01]$.

Accuracy: Summary. Age-related differences in accuracy were disproportionately larger in the dual-task condition relative to the alone condition with old participants showing reduced accuracy due to interference. Furthermore, a direct comparison between the PI and CI conditions showed that increased overlap between the individual tasks was disproportionately more deleterious for old than for young participants.

Table 5

Descriptive Statistics $(M, S E)$ for Total Correct Responses and the Change Ratio in Correct Responses per Group for the Alone, PI, and CI Conditions of the DVRT

\begin{tabular}{lccccc}
\hline & \multicolumn{2}{c}{ True Negative } & & \multicolumn{2}{c}{ True Positive } \\
\cline { 2 - 3 } \cline { 5 - 6 } & $M$ & $S E$ & & \multicolumn{2}{c}{$S E$} \\
\hline Alone & & & & \\
$\quad$ Young TC & 19.1 & .35 & & 18.1 & .43 \\
Old TC & 17.3 & .53 & & 16.6 & .54 \\
PI & & & & \\
Young TC & 19.2 & .16 & 17.9 & .43 \\
$\quad$ Young $\Delta$ ratio & .011 & .02 & -.008 & .02 \\
Old TC & 16.5 & .66 & 16.1 & .66 \\
Old $\Delta$ ratio & -.038 & .04 & -.016 & .05 \\
CI & & & & \\
Young TC & 17.9 & .52 & 16.6 & .82 \\
$\quad$ Young $\Delta$ ratio & -.058 & .02 & -.092 & .03 \\
Old TC & 12.7 & .71 & 13.1 & .73 \\
$\quad$ Old $\Delta$ ratio & -.262 & .05 & -.201 & .04 \\
\hline
\end{tabular}

Note-TC, total correct out of 20 possible responses. $n=16$ for each group. 


\begin{tabular}{|c|c|c|c|c|}
\hline \multicolumn{5}{|c|}{$\begin{array}{c}\text { Table } 6 \\
\text { CDS-Percentage of Incorrect Digit Recall }(M, S E) \text { per Group } \\
\text { in the Alone, PI, and CI Conditions }\end{array}$} \\
\hline & \multicolumn{2}{|c|}{ Young } & \multicolumn{2}{|c|}{ Old } \\
\hline & $M$ & $S E$ & $M$ & $S E$ \\
\hline Alone & 1.2 & .35 & 4.2 & .79 \\
\hline PI & .78 & .33 & 5.2 & 1.6 \\
\hline \multicolumn{5}{|l|}{ CI } \\
\hline 1 st digit set & 1.3 & .38 & 11.3 & 2.6 \\
\hline 2nd digit set & 1.4 & .36 & 10.8 & 2.6 \\
\hline
\end{tabular}

Note $-n=16$ for each group.

\section{CDS}

Descriptive statistics for the percentage of incorrect digit recall in the alone, PI, and CI conditions are presented per group in Table 6. Repeated measures ANOVAs were performed on two models with age as the between-subjects factor and task as the three-level within-subjects variable. The first model included the percentage of incorrect digit recall in the alone and PI and in the first digit set of the CI. The second model used the second digit set of the $\mathrm{CI}$ as the third-level task.

Model 1. The main effects for age $[F(1,30)=13.975$, $p<.01]$, task $[F(2,60)=9.064, p<.01]$, and the age $\times$ task interaction $[F(2,60)=7.551, p<.01]$ were statistically significant. Helmert planned contrast analyses carried out on the age $\times$ task interaction showed that the difference between young and old participants was significantly larger in the dual-task conditions, compared with the alone condition $[F(1,30)=6.640, p<.05]$. Moreover, group differences were significantly larger in the CI compared with PI $[F(1,30)=8.404, p<.01]$.

Model 2. Similar to the first model, the main effects for age $[F(1,30)=12.611, p<.01]$, task $[F(2,60)=9.409$, $p<.01]$, and the age $\times$ task interaction $[F(2,60)=7.609$, $p<.01]$ were significant. Helmert planned contrast analyses, carried out on the age $\times$ task interaction, showed that the difference between young and old participants was significantly larger in the dual-task conditions relative to the alone condition $[F(1,30)=5.867, p<.05]$. Moreover, age differences were significantly larger in the $\mathrm{CI}$, compared with the PI $[F(1,30)=9.911, p<.01]$.

The descriptive CDS data clearly showed that old participants' recall accuracy was reduced in the CI condition, whereas young participants performed equally well across task conditions. However, the near-ceiling performance by young participants might limit the interpretation of the age $\times$ task interaction. To address this analytic concern, the repeated measures ANOVAs were rerun within each age group. As expected, the effect of task for young participants was not significant for either Model 1 $[F(2,30)=.870, p=$ n.s. $]$ or Model $2[F(2,30)=1.24$, $p=$ n.s.]. Whereas for old participants, the effect of task was significant for both Model $1[F(2,30)=8.685, p<$ $.01]$ and Model $2[F(2,30)=8.843, p<.01]$.

CDS performance: Summary. Old participants incurred dual-task costs that were significantly larger than those shown by young participants. Moreover, compared with their performance in PI, old participants demonstrated a greater reduction in digit recall accuracy in both the first and second digit sets of the CI than that shown by young participants. These findings provided evidence of the sensitivity of encoding and arguably output to interference in aging.

\section{GENERAL DISCUSSION}

The present experiments were designed to evaluate whether the aging process compromises executive control of working memory. Although different notions of executive function are available, we chose to examine this issue using a dual-task paradigm that is conceptually similar to a well-established working memory model (Baddeley, 1996, 2001; Baddeley \& Hitch, 1974). The individual working memory tasks were designed to be consistent with the visuospatial sketchpad and phonological loop slave systems. Executive control was conceived as the ability to monitor and allocate attentional resources to competing task demands and was operationalized in terms of the costs incurred when performing the two tasks concomitantly. However, the present experiments differed from previous research in that the extent of temporal overlap between the two individual working memory tasks was experimentally manipulated. This was done to prevent participants from interlacing between tasks, which can minimize dual-task performance costs. Specifically, these experiments were designed to address three important issues in evaluating the effect of aging on dual-task performance.

First, it was of interest to examine whether using single tasks that represented different perceptual modalities insulated against the effect of aging on dual-task performance. Although age-related dual-task costs have been documented in a large body of literature (for a recent metaanalysis, see Verhaeghen et al., 2003), some have suggested that such costs could be reduced (Hartley \& Little, 1999) or eliminated (Baddeley et al., 2001) if the two tasks were processed via separate perceptual modalities. Findings in the present experiments consistently demonstrated that the deleterious effect of aging on dual-task performance remained in a paradigm in which the individual working memory tasks were designed to represent two separate stimulus response channels (i.e., visual-manual and auditory-verbal modalities). The high level of accuracy that old participants demonstrated on the DVRT when performed alone, in both experiments, argues against the notion that the dual-task costs they incurred were attributable to compromised mastery of the individual tasks. Furthermore, proportional transformation of the accuracy data did not eliminate the negative effect of aging on dual-task performance accuracy.

Experiment 1 showed age-related dual-task costs in reaction time that were disproportionate to group differences at baseline. However, these findings were attributed 
to the young participants' faster reaction time in the PI and CI conditions compared with the DVRT alone. Whereas positive interference was reported elsewhere for reaction time (Donk \& Sanders, 1989) and accuracy (Gick, Craik, $\&$ Morris, 1988), it was necessary to rule out if not understand its underlying causes in the context of this study. One possibility was that young participants' faster reaction time in the dual-task conditions was attributable to the test order effect in Experiment 1, which might have been related to age. However, this explanation is inconsistent with the results of Experiment 2, in which the test order effect was eliminated but the positive dual-task interference for young participants was replicated. In contrast to their performance in Experiment 1, old participants were significantly slower in the CI condition compared with the baseline condition in Experiment 2. Moreover, the age-related dual-task costs in the second experiment remained after proportional transformation of the reaction time data. These findings are consistent with Kramer, Hahn, and Gopher (1999) in suggesting that age-related differences in dual-task performance are attributable to the effect of aging on executive control and not to general slowing (Salthouse, 1985, 1996).

We can speculate that age-related differences in arousal might explain the improved performance exhibited by young participants in the dual-task conditions. Matthews and Davis (2001) and Matthews and Westerman (1994) found that increased arousal was related to performance in dual-task conditions but not single-task conditions. If one assumes that old participants were already close to or at maximal arousal in the DVRT alone condition but that young participants' arousal increased in the dual-task conditions, young participants' increased level of arousal might have been related to their faster reaction time.

\section{The Effect of Temporal Overlap}

Direct comparison of DVRT performance in the PI and CI conditions addressed the question of whether extending the degree of temporal overlap between the two single working memory tasks affected dual-task performance differentially in young and old individuals. Accuracy and reaction time performance indices from both experiments showed that old participants incurred disproportionately greater dual-task costs in the CI than in the PI condition relative to their young counterparts. These findings provide converging evidence in support of the notion that increased temporal overlap in a dual-task situation is more deleterious for old than young persons.

It is noteworthy that while the individual tasks in the two dual-task conditions remained unchanged, the transition between digit sets may have added a brief executive demand to the CI condition and thus contributed to the effect of increased temporal overlap on age-related dual-task performance. However, this brief executive demand, if significant, should be viewed in the context of a 24-sec trial.

The deleterious age-related effect of increased temporal overlap on dual-task performance is theoretically impor- tant. Extending the degree of overlap between the individual tasks represents an additional challenge to cognitive capacity that is presumably under the control of the central executive. Specifically, findings from the present experiments suggest that whereas retention appears to be rather "resistant" to interference, extending the overlap to encoding and output operations places greater demands on the central executive. Managing this increase in demands appears to be compromised by aging.

\section{Sensitivity of Encoding and Output Mechanisms to Interference in Aging}

Comparing the CDS performance between the PI and $\mathrm{CI}$ conditions provided evidence regarding the age-related sensitivity of encoding and likely output to interference. In the CI condition, encoding of the first digit set and the visual stimuli occurred simultaneously. In the PI condition, the encoding of digits occurred during retention of the visual stimuli. In the first digit set of the CI condition and in the PI condition, the recall/output of digits occurred during the retention phase of the visual task. Thus, encoding demands varied whereas output demands remained constant. The decrease in accuracy in the first digit set of the CI compared with the PI condition was greater in older participants, suggesting that the sensitivity of encoding to interference increases with age.

In the PI condition and the second digit set of the CI condition, digit encoding occurred during the retention phase of the DVRT. Recall of the second digit set in the CI condition occurred while simultaneously making a decision about the visual probe, whereas the recall of digits in the PI condition occurred during the retention phase of the DVRT. Thus, CDS output conditions varied whereas encoding demands were similar. The results indicate that concurrent output produced more age-related digit recall errors than did overlap between retention and output. Training ensured that recall of the first digit set terminated prior to the encoding of the second digit set in the CI condition. However, proactive interference might have also contributed to the reduced age-related accuracy observed on the second digit set in the CI condition.

In conclusion, ensuring that the individual tasks represent different perceptual modalities is important but insufficient when evaluating the effect of aging on how the central executive manages competing task demands. Executive control is a dynamic and task-dependent process that is sensitive to both the degree of temporal overlap and the vulnerability of specific memory mechanisms to interference. Furthermore, it may be argued that both encoding and output operations require greater involvement of the central executive, compared with retention. This view of how the central executive monitors and allocates resources to competing tasks may be generalized beyond the current paradigm because it appears to be consistent with the flexible executive system that was developed to model PRP findings (e.g., Glass et al., 2000; Meyer \& Kieras, 1997). 


\section{REFERENCES}

Anderson, N. D. (1999). The attentional demands of encoding and retrieval in young and older adults: 2 . Evidence from secondary task reaction time distributions. Psychology \& Aging, 14, 645-655.

Anderson, N. D., Craik, F. I. M., \& NaVeh-BenJamin, M. (1998). The attentional demands of encoding and retrieval in younger and older adults: 1. Evidence from divided attention costs. Psychology \& Aging, 13, 405-423.

BADDELEY, A. [D.] (1996). Exploring the central executive. Quarterly Journal of Experimental Psychology, 49A, 5-28.

BADDELEY, A. D. (2001). Is working memory still working? American Psychologist, 56, 851-864.

BadDeley, A. D., BadDeley, H. A, Buck, R. S., \& Wilcock, G. K. (2001). Attentional control in Alzheimer's disease. Brain, 124, 14921508 .

Baddeley, A. D., Bressi, S., Della Sala, S., Logie, R., \& Spinnler, H. (1991). The decline of working memory in Alzheimer's disease: A longitudinal study. Brain, 114, 2521-2542.

BADDEley, A. D., \& Hitch, G. (1974). Working memory. In G. H. Bower (Ed.), The psychology of learning and motivation (Vol. 8, pp. 47-89). New York: Academic Press.

Baddeley, A. D., Logie, R., Bressi, S., Della Sala, S., \& Spinnler, H. (1986). Dementia and working memory. Quarterly Journal of Experimental Psychology, 38A, 603-618.

Cohen, J., MacWhinney, B., Flatt, M., \& Provost, J. (1993). PsyScope: An interactive graphic system for designing and controlling experiments in the psychology laboratory using Macintosh computers. Behavior Research Methods, Instruments, \& Computers, 25, 257-271.

Crossley, M., \& HiscocK, M. (1992). Age-related differences in concurrent task performance of normal adults: Evidence for a decline in processing resources. Psychology \& Aging, 7, 499-506.

Donk, M., \& SANDERS, A. F. (1989). Resources and dual-task performance: Resource allocation versus task integration. Acta Psychologica, 72, 221-232.

FISK, A. D., \& FISHER, D. L. (1994). Brinley plots and theories of aging: The explicit, muddled and implicit debates. Journal of Gerontology, 49, P81-P89.

Gick, M. L., Craik, F. I. M., \& Morris, R. G. (1988). Task complexity and age differences in working memory. Memory \& Cognition, 16 353-361.

Glass, J. M., Shumacher, E. H., Lauber, E. J., Zurbriggen, E. L., Gmeinkl, L., Kieras, D. E., \& Meyer, D. E. (2000). Aging and the psychological refractory period: Task coordination strategies in young and old adults. Psychology \& Aging, 15, 571-595.

Hartley, A. A. (1992). Attention. In F. I. M. Craik \& T. A. Salthouse (Eds.), Handbook of aging and cognition (pp. 1-49). Hillsdale, NJ: Erlbaum.

Hartley, A. A. (2001). Age differences in dual-task interference are localized to response-generation processes. Psychology \& Aging, 16, 47-54.

HaRTLEY, A. A., \& LittLe, D. M. (1999). Age-related differences and similarities in dual-task interference. Journal of Experimental Psychology: General, 128, 416-449.

KRAMER, A. F., HAHN, S., \& GoPHER, D. (1999). Task coordination and aging: Explorations of executive control processes in the task-switching paradigm. Acta Psychologica, 101, 339-378.

Li, K. Z. H., Lindenberger, U., Freund, A. M., \& Baltes, P. B. (2001). Walking while memorizing: Age-related differences in compensatory behavior. Psychological Science, 12, 230-237.

Matthews, G., \& Davis, R. D. (2001). Individual differences in energetic arousal and sustained attention: A dual-task study. Personality \& Individual Differences, 31, 575-589.
Matthews, G., \& Westerman, S. J. (1994). Energy and tension as predictors of controlled visual and memory search. Personality \& Individual Differences, 17, 617-626.

MATTIS, S. (1988). Dementia Rating Scale (DRS). Odessa, FL: Psychological Assessment Resources.

McDowd, J. M., \& SHAw, R. J. (2000). Attention. In F. I. M. Craik \& T. A. Salthouse (Eds.), Handbook of aging and cognition (2nd ed., pp. 221-292). Mahwah, NJ: Erlbaum.

Meyer, D. E., Glass, J. M., Mueller, S. T., Seymour, T. L., \& Kieras, D. E. (2001). Executive-process interactive control: A unified computational theory for answering 20 questions (and more) about cognitive ageing. European Journal of Cognitive Psychology, 13, 123-164.

Meyer, D. E., \& Kieras, D. E. (1997). A computational theory of executive cognitive processes and multiple-task performance: Part 2. Accounts of psychological refractory-period phenomena. Psychological Review, 104, 749-791.

NeLSON, H. E. (1982). National Adult Reading Test: Test manual. Windsor, U.K.: NFER Nelson.

Nyberg, L., Nilsson, L.-G., OlofsSon, U., \& BäckMAN, L. (1997). Effects of division of attention during encoding and retrieval on age differences in episodic memory. Experimental Aging Research, 23, 137143

PAshler, H. (1994). Dual-task interference in simple tasks: Data and theory. Psychological Bulletin, 116, 220-224.

Perfect, T. J. (1994). What can Brinley plots tell us about cognitive aging? Journal of Gerontology, 49, P60-P64.

Salthouse, T. A. (1985). A theory of cognitive aging. Amsterdam: North-Holland.

SAlthouse, T. A. (1996). The processing speed theory of adult age differences in cognition. Psychological Review, 103, 403-428.

Salthouse, T. A., Rogan, J. D., \& Prill, K. A. (1984). Division of attention: Age differences on a visually presented memory task. Memory \& Cognition, 12, 613-620.

SoMBERG, B. L., \& SALTHOUSE, T. A. (1982). Divided attention abilities in young and old adults. Journal of Experimental Psychology: Human Perception \& Performance, 8, 651-663.

Spreen, O., \& STrauss, E. (1998). A compendium of neuropsychological tests: Administration, norms, and commentary. New York: Oxford University Press.

STERnBerG, S. (1966). High-speed scanning in human memory. Science, 153, 652-654.

STERNBERG, S. (1969). The discovery of processing stages: Extensions of Donders' method. Acta Psychologica, 30, 276-315.

Tun, P. A., \& Wingfield, A. (1994). Speech recall under heavy load conditions: Age, predictability and limits on dual-task interference. Aging \& Cognition, 1, 29-44.

Verhaeghen, P., \& Cerella, J. (2002). Aging, executive control, and attention: A review of meta-analyses. Neuroscience \& Biobehavioral Reviews, 26, 849-857.

VerHAEGHEN, P., MARCOEN, A., \& Goossens, L. (1993). Facts and fiction about memory aging: A quantitative integration of research findings. Journal of Gerontology, 48, P157-P171.

Verhaeghen, P., Steitz, D. W., Sliwinski, M. J., \& Cerella, J. (2003). Aging and dual-task performance: A meta-analysis. Psychology \& Aging, 18, 443-460.

WECHSLER, D. (1997). Wechsler Adult Intelligence Scale-3rd Ed. San Antonio: Psychological Corporation.

Whiting W. L., \& Smith, A. D. (1997). Differential age-related processing limitations in recall and recognition tasks. $\underline{\text { sychology } \&}$ Aging, 12, 216-224.

Wickens, C. D., Braune, R., \& Stokes, A. (1987). Age differences in the speed and capacity of information processing: 1. A dual-task approach. Psychology \& Aging, 2, 70-78. 
APPENDIX

Visual Stimuli: Examples of Trials That Vary in Terms of Their Mean Pixel Ratios

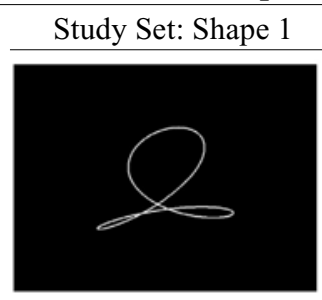
Study Set: Shape 2 Probe
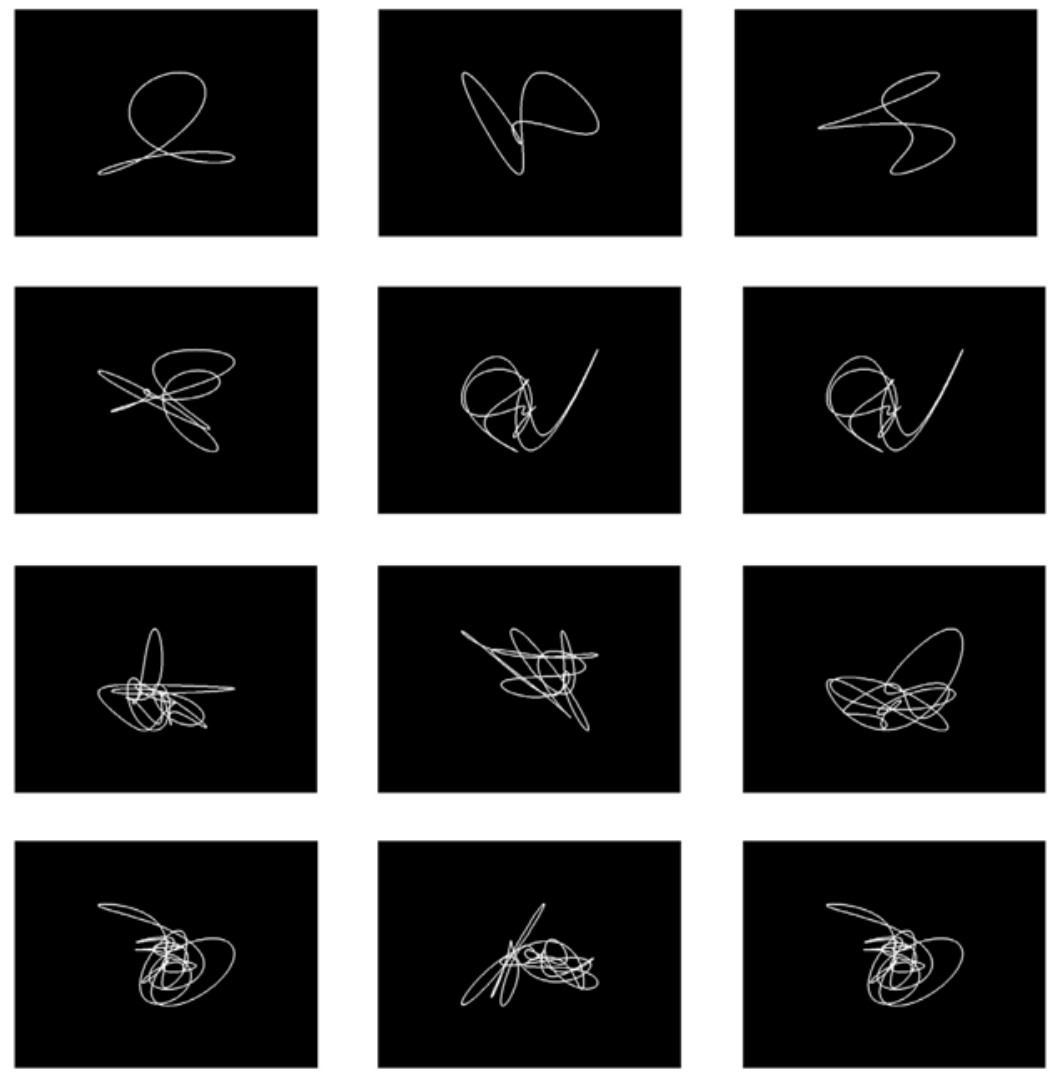

Note-True negative trials are represented in Lines 1 and 3. True positive trials are represented in Lines 2 and 4.

(Manuscript received February 3, 2003;

revision accepted for publication April 15, 2004.) 